\title{
Effect of the geotextiles reinforcement area on the stabilization of soil deformations in Chlef city (Algeria)
}

\author{
Zohra Boutaraa $^{1 * *}$, Ahmed Arab ${ }^{l}$ and Mohammed Chemmam ${ }^{1}$ \\ 1 Laboratoire des Science des Matériaux et de l'Environnement, Université HBB de Chlef, 02000 Chlef, Algérie
}

\begin{abstract}
This communication presents a laboratory study on the effect of the geotextiles reinforcement area on the improvement of the mechanical proprieties of a sandy soil, especially on the stabilization of soil deformations that affect road pavement in Chlef city (Algeria), which is a prone area to seismic risk. The study is carried out via a monotonic undrained tests using the triaxial apparatus. Reconstructed soil samples having a relative density corresponding to two soil states: loose $(D r=20 \%)$ and medium dense $(D r=50 \%)$ are tested. The aim of the study is to highlight the improvement that can bring the increase of the area of geotextile reinforcement on the behavior of the studied soil. Results show that the reinforcement area has a great positive effect on the increase of the soil resistance, traduced by the stress deviator and by the friction angle, and on the decrease of the pore pressure that reduce the liquefaction risk.
\end{abstract}

\section{Introduction}

Soil reinforcement technics represent an efficient tool that can be used in the aim to resolve instability problems encountered in low mechanical quality soils, especially in prone area to seismic risk. These technics that have been developed considerably all over the world are actually used as an incorporate part of civil engineering projects since it can increase the soil mechanical properties and give it a better behavior, particularly in seismic zones, where there are used as a seismic risk management tool. Among soil reinforcement process frequently used we are interested by geotextiles, which consists in introducing in the soil one or more layer number of geotextiles. This process gives very good results in superficial layers of sandy soils, where the confinement has low values [1]. Several researchers have studied the reinforcement of Chlef sandy soil using geotextiles. However their interest pointed on the effect of the increase of geotextiles layers number on the soil mechanical improvement [2, 3]. In Chlef city, the road pavement of the main express national road (RN4) that longe Chllif river is instable and important deformations are constantly emerging (Fig.1). These deformations are due to the intensive dynamic load supported by the pavement and to the low soil quality which is an alluvial quaternary deposit with low compactness, mainly formed of sand, gravel and clay and which superficial layers have low shear wave propagation ( $\mathrm{Vs}=230 \sim 350 \mathrm{~m} / \mathrm{s})$, and to the high settlement potential in this section [4]. Note that Chlef city is located in a high seismic zone [5] and since the soil saturation degree is sufficiently high in these layers adjacent to Chellif river, the liquefaction risk is also important in case of a seismic event. Therefore, in this work our interest is oriented on the effect of the increase of the geotextiles reinforcement area on the behavior of this soil and we propose to study the effect of the geotextiles reinforcement area on the undrained behavior of Chlef sandy soil under monotonic loading.

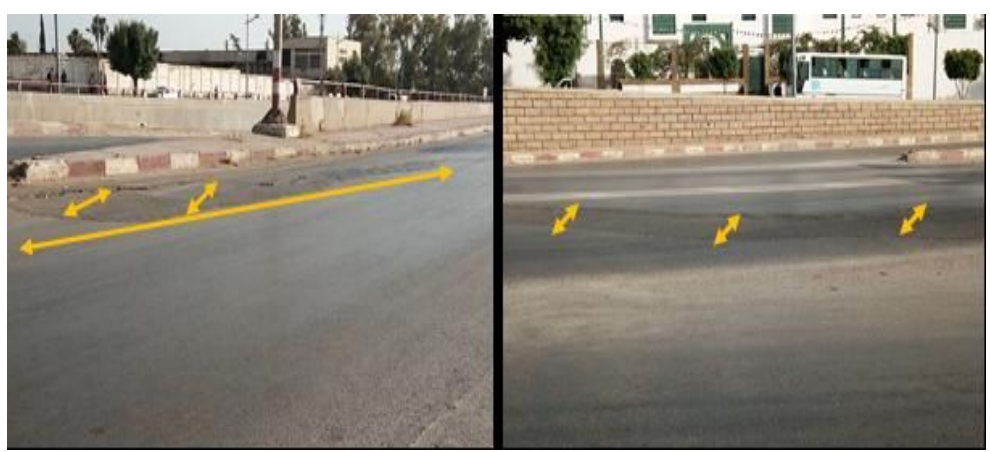

Figure 1: Important deformations of a section of RN4 express road in Chlef city (Algeria)

\footnotetext{
Corresponding author : z.boutaraa@univ-chlef.dz
} 


\section{Experimental Procedure and materials}

A laboratory study is carried out using the triaxial apparatus in undrained monotonic conditions. Soil samples, constituted by Chlef sand $\left(\mathrm{\gamma}=2.69 \mathrm{~g} / \mathrm{cm}^{3}\right)$ and $20 \%$ of silt $\left(\mathrm{\gamma}=2.70 \mathrm{~g} / \mathrm{cm}^{3}\right.$, ) are reconstituted using under-compaction method [6] and saturated before tested. The saturation degree affecting significantly the soil liquefaction resistance is evaluated by measuring the Skempton's coefficient B after consolidation [7]. Two relative densities ( $\mathrm{Dr}=20$ and $50 \%$ ) corresponding to two soil states (loose and medium dense) are experimented.

The reinforcement is made using a geotextile type NOTEX GX 100/50 which characteristics are given in Table 1. One geotextile layer is placed at the sample middle high (Fig.2).Three samples are tested for every soil state: a first sample without reinforcement (SR), a second one in which the reinforcement géotextile area is equal to $2 / 3$ of the sample section $(2 / 3 \mathrm{~S})$ and a last one in which the reinforcement géotextile area is equal to the totality of the sample section (S). Note that these géotextile areas are chosen because of the easiness of their cutting in the laboratory and positioning in the sample.

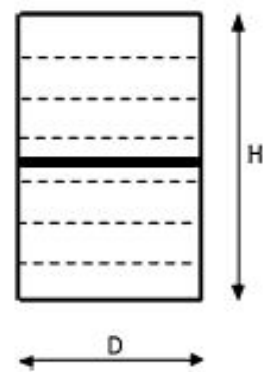

Figure 2: Position of the geotextiles in the soil samlpe

Table 1: Technical characteristics of the used geotextile

\begin{tabular}{|l|l|c|c|}
\hline \multicolumn{1}{|c|}{ Characteristics } & \multicolumn{1}{c|}{ Norm } & Value & Tolerance \\
\hline Mass per unit area (g/m2) & Standard ISO 9864 & 300 & $\pm 10 \%$ \\
Thickness under 2 kPa (mm) & Standard 964-1 & 1,25 & $\pm 20 \%$ \\
Surface porosity (\%) & & 43 & \\
Tensile rupture strength (kN/m) & Standard EN ISO10319 & SP : 100 & Min. Val \\
& & ST : 50 & Min. Val \\
Tensile strength at 2\% strain SP (kN) & & 16 & \\
Tensile strength at 3\% strain SP (kN) & & 23 & \\
Tensile strength at 5\% strain SP (kN) & & & Val. Min. \\
Strain at failure in tension (\%) & Standard EN ISO10319 & 38 & $\pm 20 \%$ \\
& & & $\pm 20 \%$ \\
Dynamic perforation (mm) & Standard EN ISO13433 & 19 & $\pm 20 \%$ \\
Static puncture CBR (KN) & Standard EN ISO12236 & 2,5 & $-10 \%$ \\
\hline
\end{tabular}

\section{Study results}

\subsection{Effect of the geotextile reinforcement area on the soil resistance}

Figure 3a shows the evolution of the stress deviator $q$ in the loose soil samples before and after its reinforcement. It is concluded that the reinforcement area affect considerably the soil resistance since the maximum value of $q$ reaches 94 $\mathrm{kPa}$ in the no reinforced sample, then rises to the values of 120 and $186 \mathrm{kPa}$ in the reinforced samples on an area of $2 / 3$ and of the total sample section, respectively. Figure $3 \mathrm{~b}$ shows the pore pressure $U$ evolution in the no reinforced sample reaching maximal values of 190 corresponding to an axial deformation $\varepsilon_{l}$ of $5 \%$, then $U$ decreases linearly until $140 \mathrm{kPa}$. $U$ maximal value in the samples which are reinforced on an area equal to $2 / 3$ and to the totality of the sample section are less important: 85 and $80 \mathrm{kPa}$, respectively. In this latter sample, the maximum axial deformation $\varepsilon_{l}$ is equal to $3 \%$, and the decrease of $U$ is more pronounced until its annulation. 

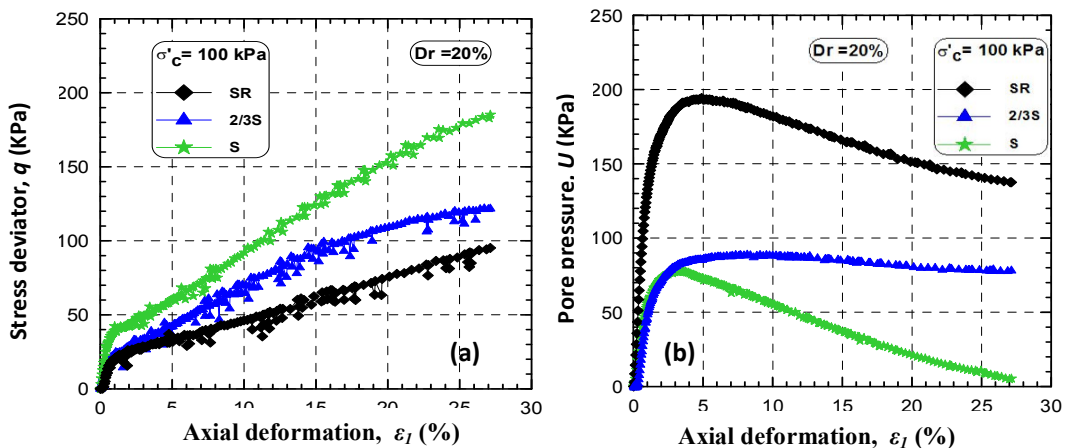

Figure3: Reinforcement area effect on the undrained behavior of the loose soil sample:

(a) Evolution of the stress deviator and (b) Evolution of the pore pressure

In the case of the medium dense samples, the increase of the geotextile reinforcement area effect becomes more pronounced. Indeed, the maximum value of $q$ that do not exceed $150 \mathrm{kPa}$ in the no reinforced sample, rises the values of 180 and $200 \mathrm{kPa}$ in the samples reinforced on the $2 / 3$ and on the totality of their sections, respectively (Fig. 4a). It can be observed that the second sample has initially a dilatant behavior and has a higher resistance than the last one (reinforced on its total section) until a $\varepsilon_{l}$ value of $15 \%$. After this a different behavior is observed; an increase of the sample resistance versus of the reinforcement area, which is due to the geotextile role that deprives radial deformations in the sample and consequently the soil has contracting behavior. Concerning the pore pressure evolution, it can be observed that the reinforcement area has an important effect on its decrease. Indeed, for about a value of $2.5 \%$ of $\varepsilon_{l}, U$ reaches maximal values of 79, 75 and $72 \mathrm{kPa}$ in the no reinforced sample, the reinforced on an area of $2 / 3$ and on the totality of the sample section, respectively (Fig. 4b).
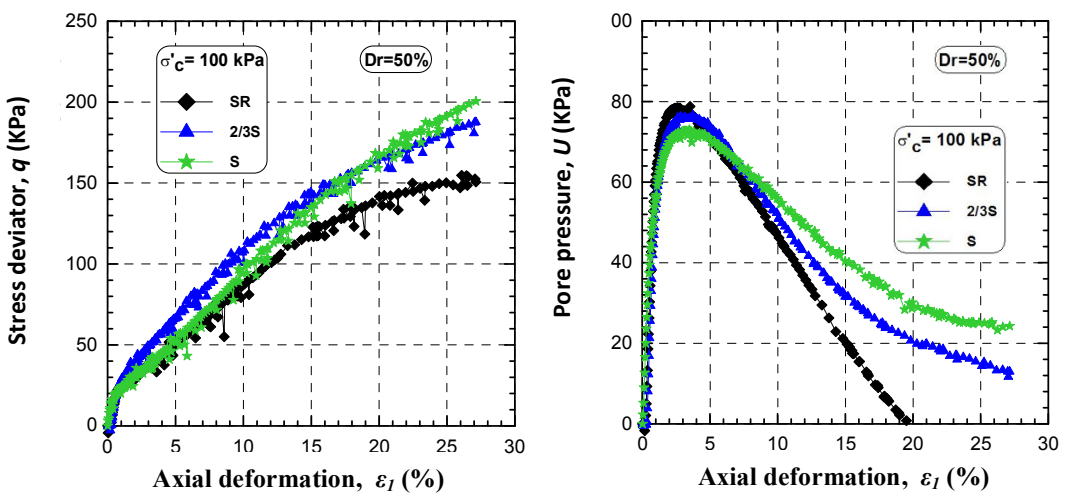

Figure 4: Reinforcement area effect on the undrained behavior of the medium dense sample:

(a) Evolution of the stress deviator and (b) Evolution of the pore pressure

\subsection{Effect of the geotextile reinforcement area on the soil friction angle}

The introduction of geotextile reinforcement in the sample conducts to a modification of the soil mechanical characteristics. Among these characteristics we are interested by the friction angle $\varphi$ (eqt 1 ) because it is the most important one that affect the behaviour of sandy soils as these encountered in the studied area. Other parameters can be measured and highlighted as the secant modulus for instance, but due to the importance of the friction angle and the deviator stress that characterize the resistance of the studied soil, we discussed just these two parameters in this paper.

$$
\varphi=\operatorname{Arcsin}(3 * \eta / 6+\eta)
$$

Where $\boldsymbol{\eta}$ is given by equation 2 :

$$
\eta=q / \sigma^{\prime} e f f
$$


Where $q$ is the deviator stress and $\sigma^{\prime}$ eff is the effective stress.

As an example in the medium dense sample, this evolution is exponential. Since $\varphi$ value is equal to $26.9^{\circ}$ in the no reinforced sample then it becomes equal to $28.47^{\circ}$ and to $29.26^{\circ}$ in the samples reinforced on the $2 / 3$ and on the totality of their sections, respectively (Fig.5). This result confirms the positive effect of the increase of the geotextile reinforcement area on the mechanical behavior of the studied soil.

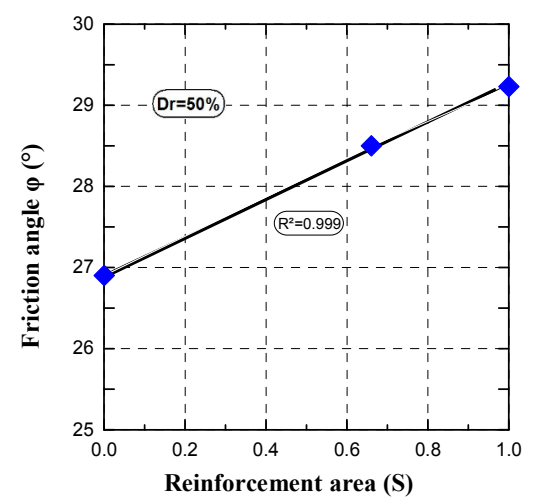

Figure 5: Reinforcement area effect on the soil friction angle

\section{Conclusions}

This communication gives the results of a laboratory study on the effect of the geotextiles reinforcement area on the improvement of the mechanical proprieties of Chlef sandy soil. The soil samples are tested under undrained monotonic conditions using the triaxial apparatus and for two relative densities corresponding to a loose and medium dense soil states. The used reinforcement areas are equal to $2 / 3$ and to the totality of the sample section. The obtained results show that the increase of the geotextiles reinforcement area has a positive effect on the improvement of the mechanical properties of this soil after reinforcement. This improvement affects the soil resistance traduced by the stress deviator $q$ and the soil friction angle $\varphi$. In another hand, the decrease of the pore pressure $U$ indicates the diminution of the liquefaction risk that can occurs during a seismic event in Chlef city (Algeria) which is a prone area to seismic risk. The solution proposed in this communication to resolve soil deformations affecting the main express national road (RN4) in Chlef city, can easly be applied on real scale using geotextiles layer that will be placed under the road pavement on a depth of about $0.70 \mathrm{~m}$ on all the area affected by vertical or horizontal deformations.

\section{References}

1. Arab A., Belkhatir M., Sadek M. Geot. Geol. Eng. 34, 347-358 (2016).

2. Benessalah I., Arab A., Villard P. et al. Geot. Geol. Eng. 34, 6: 1775-1790 (2016)

3. Brahim AEK., Arab A., Belkhatir M., Shahrour I. J. Earth Sci. 27, 6: 1060-1070 (2015)

4. Woodward-Clyde Consultants. Microzonation Sismique d'Echllif - Algérie. Alger, p 166 (1984)

5. RPA. Règles Parasismiques Algériennes. Centre National de Recherche Appliquée en Génie Parasismique, Alger (2003)

6. Ladd R. S. Geot. Tes. J, ASTM 1(1): 16-23 (1978).

7. Skempton AW. Géot. Vol.4,4 : 143-147 (1954) 